Nature Tourism and Mental Health: Parks, Happiness, and Causation.

Ralf Buckley

International Chair in Ecotourism Research (Emeritus)

ralf.c.buckley@gmail.com, r.buckley@griffith.edu.au

$+61417711502$

Word count: 9138 total

Acknowledgements. Ethics protocol \#2017/838. 


\title{
Nature Tourism and Mental Health: Parks, Happiness, and Causation.
}

\begin{abstract}
Park tourism is correlated with better mental health, with high economic value, but some aspects of causal mechanisms have remained unresolved. I interviewed 238 outdoor nature and adventure tourists of all ages and backgrounds, at subtropical national parks in Queensland, Australia. I asked whether they visit parks because they are happy, or they are happy because they visit parks. A large majority, $82 \%$, picked the latter. Of those asked, $87.5 \%$ reported short-term emotional benefits; $60 \%$, medium-term recovery from stress; and $20 \%$, long-term changes in worldview. Research priorities include: economic scale; experiential components and commercial design for different market segments; and tests of mental health benefits for tourism more generally.
\end{abstract}

Keywords: wellbeing; wildlife; satisfaction; experience; happiness

\section{Introduction}

There are significant interrelationships between nature, tourism, and mental health (Bratman et al., 2019; Buckley et al., 2019; Holland et al., 2018; Thomsen et al., 2018), but these relationships are not all straightforward. Various prior lines of research have provided scientific support for existing social patterns favouring outdoor recreation (Holland et al., 2018; Thomsen et al, 2018). Such research has also suggested likely directions for future social change, notably the formal adoption of nature therapies in mainstream medicine, and the potential delivery of such therapies by nature tourism enterprises (Buckley, 2019). There are, however, still some gaps in the matrix of links between the three major components.

This contribution addresses one of those gaps. Does taking part in outdoor nature, adventure and wildlife tourism and recreation make people happier and improve their mental health; or, are happier and healthier people more likely to devote their discretionary time and resources to outdoor tourism and recreation? Does the mental health of individuals change over time as a result of nature tourism, or is there simply a section of the population that enjoys good mental health and also engages in nature tourism? More concisely: are people happy because they visit parks, or do they visit parks because they are happy? This is a fine distinction, but an important one, for reasons considered below.

People who visit parks include nature tourists and local residents. Tourism, recreation, and visitation are defined differently in different countries. Park visitors are classified as tourists if they meet any of three conditions. The first is if they visit a park as a client of a commercial tourism enterprise. The second is if they travel $>40 \mathrm{~km}$ from home, or stay away from home overnight, in order to visit the park. The third is if they are on holiday as a tourist, and visit a park as part of that holiday, even if the park is $<40 \mathrm{~km}$ from their holiday accommodation. Except for local residents visiting local parks, therefore, most park visitors are nature tourists.

The principal lines of research to date, linking nature, tourism and mental health and happiness, may be summarized as follows (Bratman et al., 2019). First, large-scale geographical pattern analyses, and longitudinal panel studies, have shown that people who live near green or blue space generally enjoy better mental health than those who do not, even after controlling for socioeconomic factors (Alcock et al., 2014; Song et al., 2018; White et al., 2016, 2019; Wyles et al., 2017). 
Second, small-scale experimental studies have shown that individuals experiencing various forms of short-term and low-key exposures to components of nature, demonstrate improved mental health and happiness, relative to control individuals (Bratman et al., 2019; Buckley et al., 2018, 2019; Frühauf et al., 2016; Frumkin et al., 2017; Larson, Jennings, \& Cloutier, 2016; Shanahan et al., 2016; Terraube, Fernández-Llamazares, \& Cabeza, 2017; TwohigBennett \& Jones, 2018; Wang et al., 2017). Multiple parameters have been examined.

Third, numerous studies of outdoor recreation indicate that most participants perceive themselves as gaining improved self-perceived personal well-being (Coghlan, 2015; Lengieza et al., 2019; Smith \& Diekmann, 2017; Uysal et al., 2016) through their preferred activities (Groulx et al., 2016; Hardiman \& Burgin, 2017; Holland et al., 2018; Kim et al., 2015; Stone \& Stone, 2017; Thomsen et al., 2018). This includes tourism in parks and forests (Azara et al., 2018; Kim et al., 2015; Ohe et al., 2017; Puhakka, Pitkänen, \& Siikamäki, 2017; Young, McGrath, \& Adams, 2018; Xiao et al., 2017; Xu et al., 2014). It also includes transformative effects (Brymer \& Schweitzer, 2013; Wolf, Ainsworth, \& Crowley, 2017; Wolf, Stricker, \& Hagenloh, 2015), and mental health specifically (Holland et al., 2018; Thomsen et al., 2018).

The mediating roles of place attachment (Kil et al., 2012; Ramkisson, Smith, \& Weiler, 2013) and connectedness to nature (Groulx et al., 2016), and the effects of visit frequency (Choe, Schuett \& Sim, 2017; Hardiman and Burgin, 2017) and of cultural differences (Puhakka et al., 2017; Stone \& Stone, 2017; Xiao et al., 2017; Xu, Sofield \& Li, 2104) have also received attention. There is one study (Levi et al., 2018) which showed that for individuals suffering from clinically diagnosed depression, voluntarily taking part in commercial tours led to improvements for some patients. That study, however, was not related to parks or nature tourism. In the link between discretionary nature tourism and mental health, tourism research has not specifically addressed the direction of causation.

A key difference between these various methodological approaches is the degree to which different individuals: (a) express personal interests in nature-based recreational opportunities; (b) are free to take them up; and (c) actually do take them up. For large-scale geographical studies, for example, wealthier individuals can choose whether or not to live in areas with parks and vegetation, but less well-off individuals may not have that choice (Howard Centre for Investigative Journalism, 2019). Controlling for wealth and education in multiple regression studies thus relies principally on the wealthy individuals who choose to live in low-nature areas downtown, rather than less wealthy individuals who might choose to live in leafy suburbs if they could afford it.

For experimental studies, treatments and controls are allocated randomly between volunteers who have registered to take part. This is a necessary methodological feature of randomized controlled trials. In the process, however, it bypasses the preferences of individuals, for or against spending their discretionary time in nature. In social science studies of participants in outdoor tourism and recreation, researchers can enquire about respondents' individual motivations to take part, and factors contributing to experiences and satisfaction (Holland et al., 2018; Thomsen et al., 2018). Few such studies, however, also obtain comparable data from individuals who do not take part in the activities concerned (Boyd et al., 2018).

These considerations are not critical where the aim is simply to demonstrate that people can enjoy nature tourism and benefit from it. They do, however, become critical in two key circumstances. The first is in quantifying therapeutic outcomes, and their economic value, 
across entire populations (Buckley et al., 2019). The second is in the creation of prescriptible nature therapy products for delivery by outdoor tourism enterprises as part of mainstream healthcare systems (Buckley, 2019); where diagnosis, dose and duration are key components (Shanahan et al., 2015; Buckley et al., 2016, 2018). For both of these purposes, it is critical to know whether some people benefit from nature and others do not, or whether everyone can do so if given the opportunity.

The context to this question has three key components. First, in developed nations such as Australia, national statistics show that in practice $54-70 \%$ of people visit national parks, and the rest do not. Second, recent data show that individuals who visit national parks report about $3 \%$ greater personal well-being, on average, than those who do not, after controlling for demographic and socioeconomic factors, physical health and exercise, and non-park greenspace use (Buckley et al., 2019). This differential in well-being is about the same scale as the differential associated with income, so it is substantial. Third, individuals with greater wealth, mental health, and park use at early ages, retain those characteristics at later ages (Browning \& Rigolon, 2019; Buckley et al., 2019; Lee et al., 2017; Stafford et al., 2015).

Within this context, there are two competing hypotheses, each fitting all three components as above, and each equally plausible. We can distinguish between them only through new empirical data. The first hypothesis is that all individuals have potentially similar interests in nature, and all can experience similar gains in mental health and happiness through nature exposure, but only some have experienced the social circumstances that allow or encourage them to visit parks. Under this hypothesis, visiting parks makes people happy.

The second, competing hypothesis is that some people are happier than others, for reasons unrelated to park visitation, and the happier group visit parks more frequently. Under this hypothesis, happy people visit parks. Note that these hypotheses, whilst competing, are not mutually exclusive. It is possible that happy people visit parks, and visiting parks maintains or improves their happiness, creating a positive feedback which maintains or enhances differentiation between happy park visitors and unhappy non-visitors.

The distinction between these two hypotheses is ultimately a distinction between individual psychology and social equity. It determines whether or not nature-based tourism can contribute to alleviating the high individual, social and economic costs of poor mental health (Buckley et al., 2019); and in the process, create a major new commercial market for outdoor tourism enterprises (Buckley, 2019).

People who do take part in nature tourism, including park visitation, do indeed have better mental health, on average, that those who do not (Bratman et al., 2019); and this differential in mental health, and associated economic costs, confers an additional economic value on national parks worldwide, estimated at US\$6 trillion per annum (Buckley et al., 2019). If there were no opportunities for people to experience outdoor nature by visiting parks, then the costs of poor mental health in developed nations would increase from 10\% of GDP as currently, to $17.5 \%$ (Buckley et al., 2019). This value exists in any case, whether people are happy because they visit parks, or visit parks because they are happy. The opportunity to reduce costs of poor mental health by using nature tourism as therapy, however, depends on which of these two options applies.

Currently, individuals with poor mental health are treated principally with prescription pharmaceuticals and courses of psychotherapy. It has been proposed repeatedly that these 
individuals could instead receive courses of nature therapy, once diagnosis, dose and duration have been determined (Bratman et al., 2019; Buckley et al., 2018; Shanahan et al., 2016). It has also been proposed that courses of nature therapy could be constructed and delivered by outdoor tourism companies, as prescriptible and insurable healthcare products, funded by health insurance and government healthcare systems (Buckley, 2019).

That approach could only work, however, if people who currently do not spend time in nature, would: (a) gain improved mental health from spending time in nature; (b) be able to change their lifestyle preferences and priorities so as to spend time in nature; and (c) be able to overcome existing social obstacles to spending time in nature, which apply particularly to at least some social groups with high incidence of poor mental health (Boyd et al., 2018; Buckley et al., 2016). That is, these proposals would only work if nature tourism, including visiting parks, makes people happy.

The current paradigm in park tourism, recreation and visitation, however, is that people who are socially or economically disadvantaged visit parks less, or not at all (Boyd et al., 2018; Buckley et al., 2016, 2019; Warner et al., 2020; Zanon et al., 2019). This population subsector is also more likely to suffer from poor mental health. That is, the current belief amongst psychologists is that nature makes people happier; but the current belief amongst parks agencies, who provide the principal access to nature, is that happy people visit parks; or equivalently, that disadvantaged and unhappy people don't.

That is, the distinction addressed here has major implications. How, therefore, can we differentiate between the two competing hypotheses, as outlined above? Note that we are not asking here whether people who do not visit parks are, on average, less happy or healthy. We already know that. We are not asking whether people who currently do visit parks, are happier on average than those who do not. We already know that too. We are asking very specifically, whether those particular people who both (a) choose to visit parks, and (b) are happy, are happy because they visit parks, or visit parks because they are happy.

\section{Materials and Methods}

\section{Options for Testing Causation: Drawbacks \& Limitations}

Randomised controlled trials, RCTs, are used widely in research on nature exposure and mental health (Corazon et al., 2018; Jerrett et al., 2018; Stigsdotter et al., 2018). They are not always feasible: e.g., if they would require too large an intervention in individual lives (Gottfredson et al., 2015; Frieden, 2017). Thus Levi et al. (2018), testing the effects of tourism on mental health patients, were unable to use randomised controls, because patients selected and paid for their own holidays. For the current study, an RCT would need to assign members of a pre-selected group of individuals randomly to different levels or patterns of outdoor tourism, and compare their mental health parameters before and after either tourism or a control activity. This would override individual choices to take part in nature tourism or park visits, which are central to the question addressed. Hence RCTs are not applicable here.

Long-term panel studies are also used widely in analysing mental health (Dzhambov et al., 2018; Propper et al., 2005; Song et al., 2018). There are multi-year panel studies of mental health in relation to various potential causal factors, including proximity to, and time spent in, residential green and blue space (Song et al., 2018; White et al., 2016, 2019; Wyles et al., 2017). None of these, however, include nature tourism as an individual discretionary choice. 
To demonstrate direction of causation, a long-term panel study could track patterns in nature tourism or park visitation, and in mental health and happiness, for the same individuals simultaneously, for an extended period of years or decades. It would need to identify individuals whose park use and nature tourism patterns changed through time, and whose mental health and happiness also changed through time, and demonstrate that one of these two always changed first, and the other always followed. The shorter the time lag for a change in one to be followed by a change in the other, the shorter the time resolution needed to demonstrate the link. For example, if the cause and effect relationship operated over timescales of years, then a short-term study would reveal nothing. But if the causal link operates over hours, days, or weeks, then a panel study would need resampling at a similar timescale: an annual resample would be too coarse.

Currently, the timescale and lag are unknown. Most medical research on nature and mental health has used short experimental timeframes (Bratman et al., 2019). A few studies have shown benefits persisting for up to three years (Alcock et al., 2014). Recent analyses (Buckley et al., 2019) showed that even a single park visit in the previous year can generate a detectable improvement, though higher visit frequencies yield greater gains. In addition, mental health gains from a given frequency of park visits during the previous 12 months, are very similar to gains from a similar average annual frequency of visits over an individual's entire lifetime (Buckley et al., 2019). It appears, therefore, that longitudinal panel studies on this topic would need to cover periods of years, decades, or lifetimes.

Even with a long total duration, however, longitudinal panel approaches would need to repeat psychological measures at short time periods, to calculate temporally smoothed averages for comparison with frequencies of park visits or other nature tourism experiences. Smoothing is needed because measures of mental health and happiness can vary greatly over very short timescales, through a wide range of extraneous factors. Differentials between individuals in short-term (unsmoothed) measures of mental health and well-being are up to twenty times greater than the differentials due to any single factor such as exposure to nature, even after controlling for socioeconomic, demographic, and physical health factors (Buckley et al., 2019). A panel study, though a commonplace approach to causation in public health, thus has substantial drawbacks in the current case.

Given the drawbacks of RCTs and panel studies for the question addressed here, the approach adopted is through direct interviews: asking individual nature tourists visiting parks, which of the two competing hypotheses applies most accurately in their own cases. A slight variant of standard semi-structured interview techniques was used, designed to seek a smaller set of information from a larger number of time-poor individuals, each available for interview only briefly. Responses were analysed using mixed qualitative-quantitative approaches, with recoding to categorical variables (Buckley, 2018).

For the current study, the direct interview approach has fewer practical drawbacks than RCTs or panel studies. It has greater theoretical limitations, however, in the interpretation of results. It relies on individuals' abilities to identify their emotions (Buckley, 2015, 2016), and in addition, the immediate causes of those emotions. This, however, may not always be reliable, especially if multiple emotions or contributing causes apply simultaneously (Bohm \& Pfister, 2015; Tullett \& Plaks, 2016). Therefore, as for most social science analyses, the results reported here necessarily reflect respondent perceptions, not controlled experimental measurements. 


\section{Interview Variant}

Social science approaches involving detailed questionnaires or in-depth interviews require significant investment of time and attention by interviewees or respondents. Even if they receive participation incentives, this may bias the pool of subjects to those who are time-rich rather than time-poor: it is difficult to interview either shy or busy people. Person-to-person approaches can be bypassed, e.g. by using online surveys or pre-existing social media posts, but it is difficult to ensure that those provide representative samples. For population-scale analyses, online sample sizes $>100,000$ may be required, with screening and sub-selection to match sample to population on demographic and socioeconomic parameters.

Here, therefore, a slight variation was adopted, for face-to-face interviews with time-poor respondents, who were prepared to answer only one or two substantive questions. That is, the interviewee, rather than the researcher, determines the brevity of the interview. In this method, short duration is offset by increasing the number of interviews, asking different questions of different interviewees, and combining the results. That is, this method adapts the brief encounters typical of exit interviews in political polls (Atkeson \& Alvarez, 2018; Kenett et al., 2018), and the distributed comparisons used in economic choice modelling (Hensher \& Johnson, 2018). It is applicable anywhere that a large stream of potential interviewees is available, but where each one will only respond briefly. Here, it is applied to tourism in forest and beach parks.

In quantitative surveys, and also in the standard in-depth ethnographic interviews used in many qualitative approaches, each interviewee is asked about the same issues. In surveys or structured interviews, each is asked the same questions in the same order. In semi-structured interviews, the same topics are addressed in each interview, but not necessarily using the same questions, or in the same order. In qualitative approaches, interviewees may respond in great detail for some issues, and barely at all for others. For qualitative methods using constant-comparison thematic analysis (Stern \& Porr, 2017; Veal, 2017), with efficient coding and theoretical saturation (Aldiabat, 2017), this does not matter, since the aim is to extract a single aggregate pool of information and then structure it into a hierarchical knowledge framework. Unless the same dataset is also analysed using quantitative approaches (Buckley, 2018), the distribution of themes across interviewees is not considered.

In the method used here, this aspect of qualitative analysis is taken further. If it does not matter that different interviewees provide different themes that may or may not overlap, then it also does not matter if different interviewees are asked different interview questions. This is the variation in the method adopted here. As in any qualitative analysis, a set of issues was first identified, from prior published or pilot studies. Each was expressed as a single question, creating a portfolio of equal questions. Following an initial priming question to all interviewees, each interviewee was then asked only a subset of the key questions. The priming question was framed for open-length, open-text answers. Two subsequent questions were framed for closed categorical answers suitable for quantitative analysis, if sampling requirements are also met.

\section{Sites and Samples}

The interview sites were at Lamington and Springbrook National Parks in the Gondwana Rainforest World Heritage Area (forest parks), and Burleigh Heads National Park and 
associated public beaches of the Gold Coast (beach parks). Approximately equal numbers were interviewed at each. The Gold Coast, including its forest and beach parks, is a heavily marketed tourism destination with high visitation, both domestic and international. The weather is subtropical, and these sites are heavily visited year-round. Domestic visitation peaks during holiday periods. To initiate interviews, informal conversations were started with a total of $>500$ people, encountered individually at these sites, over a period of 12 months in 2018-2019. A total of 238 interviews were completed successfully. This is an order of magnitude larger than most interview studies, and two orders larger than some (Stern and Porr, 2017; Veal, 2017).

All interviewees were nature tourists (as defined earlier), voluntarily visiting the sites where interviews were conducted. The pool of interviewees is a pseudo-random sample of individuals taking part in outdoor nature-based tourism and recreation. Sampling strategies are outlined below. The interviewees are not necessarily a random or representative sample of the population as a whole, since not everyone visits parks. Individuals who do not enjoy visiting forest and beach parks, or who experience unhappiness in the outdoors, are selfselected against inclusion in the interviewee pool. Therefore, the results of these questions may not reflect broader population patterns. This is not a limitation for the current research, however, since its aim is solely to examine causation and timescale of mental health gains for individuals who do take part voluntarily in outdoor nature- and park-based tourism.

The Gold Coast region attracts over 10 million tourists annually. Most of these tourists visit either forest parks or beach parks, or both, as well as urban attractions. Therefore, a fully randomised sample was not feasible, even at an individual park. The Queensland Government parks agency does not count the numbers of visitors to its national parks. A recent estimate of tourism to Lamington NP, made by the government tourism portfolio, is $>500,000$ visitors p.a. Even if all visitors were monitored continuously, a random sample identified, and researchers waited at the trailhead full-time to request interviews, only a proportion of those approached would agree to this. Therefore, a fully randomised sample was not feasible.

The approach adopted was therefore to maximise representativeness, by carrying out interviews at all times of the day, week and year, so as to extend coverage and diversity of tourists as far as possible. Different social subgroups may visit forest and beach parks at different times of day, or for different activities. There were no social barriers to interviewing individuals both old and young, wealthy and impoverished. Each site allows for both exercise and contemplation, and interviewees included both fit and unfit individuals. There are minor cultural restrictions on who can be approached for casual conversation, but these operate via activities, not demographic or socioeconomic factors. Sites are visited by international as well as domestic tourists, and interviewees included many first languages, but this did not impose limitations for this study.

\section{Structure and Questions}

Some of the individuals encountered started conversations themselves. If not, the interviewer initiated a conversation, e.g. by offering to assist with photographs, or commenting on some aspect of the surroundings, such as weather, wildlife, scenery, clothing or equipment, or activities. If the interviewee responded, the interviewer led the conversation to the general topic of motivations for visiting parks. This allowed interviewees to comment on their reasons for using their personal leisure opportunities for activities in natural surroundings. This was the first question analysed (Q1). It also acted as a primer, to direct interviewees' 
internal attention to the principal topic of interest. This initial conversation also included a question about where interviewees were from, which is a common and acceptable topic in this region. Many local residents are identifiable from individual appearance, but since this may be unreliable, interviews were used to confirm identification as tourists.

The interviewer then led the conversation to the second set of questions (Q2), addressing one of three specific mental health outcomes derived from Buckley (2019), namely emotions, recovery and worldview. These questions were rotated between interviews, but with higher frequency for the first, as below. In each interview, the interviewer asked the interviewee whether or not that specific outcome applied in their own case. The topics were introduced in a neutral way, using the format, "some people say they feel X when they are in places like this, others say they feel not-X, what do you think?" To avoid confirmation bias, $\mathrm{X}$ and not-X were interchanged in alternate interviews. Interviewees could respond briefly or at length. If the interviewee continued with further discussion, the interviewer would also continue.

The three options for Q2 were derived as follows. Mental health outcomes from outdoor nature tourism were classified by Buckley (2019) into three tiers, using a basic open coding approach, with no axial coding. For the current study, the 6 top-tier themes were further condensed to 3, namely emotions, recovery, and worldview. It is suggested, though not critical to the current study, that these three themes can all be viewed as expressions of the same positive psychological change, over different timescales. Positive emotions are typically perceived over short timescales, seconds to minutes; psychological recovery over medium timescales, hours to days; and philosophical worldview over longer timescales, weeks to years. Note that these timescales relate to perception, not duration of memories, which can persist for many years even for short-term emotional experiences (Buckley, 2016). The three themes used in the current study are thus derived through axial coding of the 6 open-coded themes reported by Buckley (2019).

For the interviews in this study, emotions were expressed broadly as "feeling happier or generally more positive." Recovery was expressed as "feeling regenerated, more relaxed, recovered from stress". Worldview was expressed as "feeling greater clarity, or purpose, or meaning in one's life, able to see what is important." Questions were thus framed as: "some people say that [this activity] in places like this [makes them feel happier]/ [helps them recover from stress]/[etc], but others disagree - what do you think yourself?" Alternate questions were framed using opposite phraseology, such as "[less happy/more stressed]", and recategorised subsequently. Interviewees and interviewer were free to ask for clarification, if question or responses were unclear, and to continue unstructured discussion if they wished.

Once discussion on the second topic was exhausted, the interviewer asked the third and final question (Q3): "do you think that in your own case, visiting this park makes you happier, or do you think that you are more likely to visit this park when you are already happy?" This question was asked only of interviewees who had been asked about emotions in the second question, and had responded positively. Interviewees asked about recovery and worldview, and those who were asked about emotions and responded negatively, were excluded from the third question. To avoid subconscious bias, the order of clauses in the third question was reversed for alternate interviewees. Interviewees were free to respond in any way, or not at all. They could pick one response, or both, or neither, or they could discuss the topic at length, in their own words. 


\section{Consent}

During these conversations, no notes were taken, no recordings or identifications were made, and no indications were given that one of the parties involved was a researcher. Once the questions were answered, the researcher identified themselves as such, explained the reason for the research, and asked for consent to use the answers, anonymously. Conversations were continued after this consent question, if the interviewee wished. No inducements were offered. If consent was granted, the results were recorded as mobile phone voice notes, out of sight of interviewees, after the encounter was completed. This allowed other individuals to be approached subsequently, without revealing the research. If consent was not granted, the entire interaction was discarded, with no records made, since those individuals were then considered not to be part of the study at all.

A running cumulative total record of male and female respondents was also kept, to guard against any potential inadvertent bias in selection of interviewees. Discussions were discarded if the interviewees had poor English skills, or proved to be residents rather than tourists, or were reluctant to talk, or led the conversation away from the topics introduced by the interviewer, or did not grant explicit consent; or if the conversation was interrupted or conspicuously overheard by others. In practice, many interviewees proved to be greatly interested in the topic, and continued discussions with expanded scope.

This approach, conversations before consent, was adopted in order to avoid confirmation bias, whilst complying with research ethics requirements. Identifying the subject of research immediately indicates the interviewer's interests to the interviewees, predisposing them to respond in a way likely to be considered favourable. Since the interviews were conducted in parks and beaches, and since research is more commonly undertaken to test positive rather than negative propositions, identification as a researcher leads interviewees to assume that a "parks make people happy" response would be preferred. Indeed, some of the interviewees who gave the opposite response initially, began to backpedal their previous statements once the interviewer's research role was revealed. Only the initial responses were analysed here.

\section{Coding, Categorisation and Analyses}

Interviewees' responses to each of the three questions were analysed using standard thematic coding with constant comparison (Stern \& Porr, 2017; Veal, 2017). For the first question, saturation was reached very quickly, although interviews continued to include this question because of its role as a primer for the second and third questions. For those, saturation was approached more slowly, because only some interviewees gave responses beyond agreement or disagreement. Responses to the second set of questions, on mental health outcomes according to the pre-identified 3-way classification, were also coded into agreement or disagreement with each outcome addressed. Results were thus expressed as numbers or proportions agreeing or disagreeing with each of the outcomes. Differences between these proportions, and random expectations, were tested using the large-sample normal approximation to the binomial distribution.

Responses to the final question, whether visiting parks makes people happier, or happier people visit parks, were classified into 5 categories: parks first; happiness first; both at once; don't know; and other responses. The category "both at once" included two subcategories, discussed below. Results were expressed as proportions in each category. Differences between the first two categories were tested as above. Additional interviewee responses, the 
fifth category, were also analysed using thematic coding, as above. For the first question, where responses referred to a specific site, the different types of sites were compared. The second and third questions referred to outdoor nature in general, not only the specific interview sites, so comparisons between sites were not relevant for those questions.

\section{Results}

\section{Interviewees and Responses}

Of the $\sim 500$ people approached, 238 continued the conversation freely, with uninterrupted responses to all questions. The remainder either did not speak English, or were not disposed to enter conversation, or identified themselves as local residents, or led the conversation away from the topic, or gave incomplete responses, or did not grant consent to be included in the research, or were overheard or interrupted. Interviewees were $52 \%$ female, $48 \%$ male, across a wide range of age groups, excluding minors. Of those interviewed in full, all 238 were asked the first question, Q1. Of these, 112 were asked the "emotion" option in Q2; 62 the "recovery" option; and 64 the "worldview" option. Of the 112 asked the "emotion" Q2, 98 responded positively, and were then asked the "causation" Q3.

\section{Motivations}

The reasons that people gave for visiting forest and beach parks are summarised in Table 1 . There were differences in practical detail between forests and beaches, but remarkable similarity or symmetry in underlying psychology. In each case, interviewees mentioned natural attractions, outdoor activities, escape and relaxation, and social and family factors. These align well with findings of previous studies on the motivations of outdoor nature, eco and adventure tourists both generally (Arnegger et al., 2010; Kim et al., 2015), and at these sites specifically (Weaver \& Lawton, 2002).

\section{[INSERT TABLE 1 NEAR HERE]}

\section{Emotions, Recovery, \& Worldview}

Responses to the second set of questions, Q2, are summarised in Table 2. Most (87.5\%) of those asked about emotions, said that they were happy. About $60 \%$ of those asked about stress, said that they experienced recovery. And 20\% said that they had experienced a changed worldview, such as new insights, clarity or purpose in their life. Such major changes are likely to occur infrequently.

Since different interviewees were asked different questions, each in a dichotomous format, there was no formal opportunity for individuals to identify multiple outcomes. However, a number of interviewees did address multiple or overlapping outcomes in open discussion. For example, a number of interviewees linked happiness (emotion) to de-stressing (recovery), or low stress (recovery) to lifestyle (worldview). For example, they said they were happy, specifically because of relaxation; or relaxed, adding that this made them happy.

Others gave additional detail for the particular outcome addressed. For example, they commented on specific emotions, or on particular events that led to these emotions, or on particular events that led them to value recovery. A number of interviewees commented on their personal need for regular visits to forest or beach parks, either to de-stress from work, or 
for short-term escape from stressful social dynamics. Some said that their overall life philosophy or worldview was strongly tied to nature and outdoor activities, and that visits to forest or beach parks were essential to remind them of life goals and priorities.

Negative responses were relatively few, since as noted earlier, interviewees were selfselected to take part in outdoor nature tourism. A few, however, did make negative comments, eg about weather, or minor stings, bites or injuries, or group or family circumstances that led either themselves or other family members to object to the site or activity. A number also led the discussion either to their own illnesses and how they had overcome them, or to their outdoor achievements and obstacles, or to other destinations they had visited, or other aspects of their personal lives. A few said that the day's outdoor nature tourism activities had led to stress rather than recovery, for reasons such as those listed above, or because of fear of snakes, sharks, or getting washed out to sea or lost in the forest.

\section{[INSERT TABLE 2 NEAR HERE]}

\section{Causal Direction}

Results from the third question, regarding the direction of causal connection between outdoor nature-based tourism and recreation, and human mental health, may be summarised as follows. The proportions of interviewees in each of the five principal response categories are listed in Table 3. The proportion who considered that park visits lead to greater happiness $(65 \%)$ was significantly greater $(\mathrm{p}<0.001)$ than the proportion who took the opposite view $(14 \%)$. That is, of those who gave either one of these two direct responses, $82 \%$ said that nature tourism made them happy. This is the key result from this study. For the joint sample size of 78 for these two sets of responses, this indicates a $95 \%$ confidence interval of $\pm 8 \%$, i.e. $74-90 \%$, using the large-sample normal approximation to the binomial distribution.

A number of interviewees indicated that causal links operated in both directions (9\%); or were unable to determine causal direction (4\%); or gave answers that addressed different topics $(7 \%)$. Of those who answered "both" (category 3 in Table 3), there were two subcategories. Some described a long-term or lifetime process of reciprocal positive feedback, whereby visiting forest or beach parks kept them happy, and continued satisfaction with life led them to continue visiting. Others, however, said that sometimes such visits made them happier, and sometimes not, depending on circumstances (such as weather) and experiences; and in addition, that sometimes they were happy and sometimes not, for reasons unrelated to visits. That is, they identified additional complications, such that in their own cases, there was no simple causal link between nature tourism and happiness. These indicate valuable topics for future research.

In addition, a small number of interviewees gave responses that did not match any of the four predefined categories in the third question. For example, some said that particular outdoor activities, such as hiking or surfing, gave them psychological strength. This outcome has been identified previously for other adventurous outdoor activities (Brymer \& Schweitzer, 2013). Psychological strength does not match the categories in either Q2 or Q3, since it is not the same as either happiness or recovery, though it might be seen as a component of worldview. Other interviewees responded to Q3 by discussing lifestyle as a consequence of worldview. For example, some linked outdoor nature tourism and recreation to: nutritional choices, such as vegetarian diet; exercise and fitness regimes, such as yoga or running or long-distance hiking; choice of livelihood, such as jobs or businesses that allowed for flexible 
timing; or age or stage of life, such as retirement. These responses also indicate potential topics for future research.

\section{Discussion and Conclusions}

Key results may be summarised as follows. Tourists to forest and beach parks responded in very similar ways, with the same range of underlying psychological themes, though some differences in detail. The relative frequencies of positive responses for the three options in Q2, and the extended open-discussion responses from some interviewees, both fit the suggestion that positive emotions, recovery, and worldview may be viewed as different expressions of the same mental health benefit, at different timescales. Many interviewees reported short-term happiness; a moderate number reported medium-term recovery; and a much smaller proportion reported long-term worldview effects, commonly linked to lifestyle factors. This merits further research.

Whilst not derived directly from the results reported here, from a practical tourism management perspective it is worth noting that these three timescales are linked to different components of the commercial tourism industry. Single-day outdoor tours, either nature or adventure based, aim to generate customer satisfaction through short-term emotions and experiences. Nature-based tourist accommodation and associated multi-day activities, including retreats, outdoor yoga and wellness programs, ecolodges, wildlife safaris and game lodges, and multi-day outdoor adventure products can all capitalise on the recovery aspect of mental health. Backpacker tourism, grey-nomad and caravan/campervan tourism, extended cruise tourism, and similar subsectors link to the lifestyle and worldview timescale. This longer timescale also applies for tour guides in outdoor nature, eco and adventure tourism. Whilst unlikely to become as wealthy as their clients in material terms, they gain health through outdoor opportunities, despite emotional labour for their clients (Carnicelli-Filho, 2013; Mackenzie \& Kerr, 2013).

The most important finding from the current study is that whilst interviewees raised a wide variety of personal connections between outdoor tourism, nature, and mental health, the predominant direction of causation is that taking part in parks and nature tourism improves health and happiness, not the other way around. A small minority of interviewees indicated a lifetime linkage, operating in both directions simultaneously or successively. This causal direction provides strong evidence to support previous suggestions that outdoor nature and adventure tourism and recreation: (a) could be used as a tool in treating at least some clinically depressed patients (Levi et al., 2018); and (b) can contribute therapeutically to human mental health and psychological well-being (Buckley, 2019).

Indeed, the results presented here suggest that people may deliberately use outdoor nature tourism as personal mental health therapy. They choose to spend time in nature by visiting parks, and they know that this makes them happier, helps them recover from stress, and in some cases, helps improve clarity and life purpose. Future research might ask individual tourists, after they have booked nature, adventure, or wildlife holidays but before they have taken them, whether they expect an improvement in mental health, and whether that is a significant reason for making the booking.

The global estimate of $\$ 6$ trillion p.a. for the value of parks via the mental health of park tourists (Buckley et al., 2019), demonstrates an opportunity, but not a mechanism. For tourism research and practice, we need information on rates of return at much more localised 
scales. For example: what is the economic value of this mental health benefit, for different individual tourists? How can commercial tourism products best be designed and operated to as to capture this value at different timescales, for different tourism subsectors? How can mental health benefits be used in marketing, to better position outdoor nature and adventure tourism and recreation as therapeutic interventions for improved mental health? Can we identify individual tourists who perceive benefits from particular types of outdoor tourism, and are therefore more likely to purchase them? For example, these might include active or contemplative, nature or adventure, wildlife or scenery, social or solitary.

This study focussed only on outdoor tourism. For many tourists, however, various forms of indoor or urban tourism may also generate happiness and recovery. It would seem worthwhile to test whether mental health benefits may also apply across a broad range of tourism subsectors. We are accustomed to thinking of tourism as commercialised leisure and recreation. Perhaps we should also analyse tourism as a means that people use to maintain their mental health.

\section{References}

Alcock, I., White, M.P., Wheeler, B.W., Fleming, L.E., \& Depledge, M.H. (2014). Longitudinal effects on mental health of moving to greener and less green urban areas. Environmental Science and Technology, 48, 1247-1255.

Aldiabat, K.M., \& Navenec, L. (2018). Data saturation: the mysterious step in grounded theory method. Qualitative Report, 23, 245-261.

Arnegger, J., Woltering, M., \& Job, H. (2010). Toward a product-based typology for naturebased tourism: a conceptual framework. Journal of Sustainable Tourism, 18, 915-928.

Atkeson, L.R., \& Alvarez, R.M., eds. (2018). Oxford Handbook of Polling and Survey Methods. Oxford University Press, Oxford.

Azara, I., Michopoulou, E., Niccolini, F., Taff, B.D., \& Clarke, A. eds. (2018). Tourism, Health, Wellbeing and Protected Areas. CABI, Wallingford.

Böhm, G., \& Pfister, H.R. (2015). How people explain their own and others' behaviour: a theory of lay causal explanations. Frontiers in Psychology, 6, 139.

Boyd, F., White, M.P., Bell, S.L., \& Burt, J. (2018). Who doesn't visit natural environments for recreation and why: a population representative analysis of spatial, individual and temporal factors among adults in England. Landscape \& Urban Planning, 175, 102-113.

Browning, M. \& Rigolon, A. (2019). Could nature help children rise out of poverty? Green space and future earnings from a cohort in ten US cities. Environmental Research, 176, 108449.

Brymer, E., \& Schweitzer, R. (2013). Extreme sports are good for your health: a phenomenological understanding of fear and anxiety in extreme sport. Journal of Health Psychology, 18, 477-487. 
Buckley, R.C. (2015). Autoethnography helps analyse emotions. Frontiers in Psychology, 6, 209.

Buckley, R.C. (2016). Qualitative analysis of emotions: fear and thrill. Frontiers in Psychology, 7, 1187.

Buckley, R.C. (2018). Simultaneous analysis of qualitative and quantitative social science data in conservation. Society \& Natural Resources, 31, 865-870.

Buckley, R.C. (2019). Therapeutic mental health effects perceived by outdoor tourists: A large-scale, multi-decade, qualitative analysis. Annals of Tourism Research, 77, 164-167.

Buckley, R.C., Brough, P., \& Westaway, D. (2018). Bringing outdoor therapies into mainstream mental health. Frontiers in Public Health, 6, 119.

Buckley, R.C., Westaway, D., \& Brough, P. (2016). Social mechanism to get people outdoors: bimodal distribution of intertest in nature? Frontiers in Public Health, 4, 257.

Buckley, R.C., Brough, P., Hague, L., Chauvenet, A., Fleming, C., Roche, E., Sofija, E., and Harris, N. (2019). Economic value of protected areas via visitor mental health. Nature Communications, 10, 5005.

Carnicelli-Filho, S. (2013). The emotional life of adventure guides. Annals of Tourism Research, 43, 192-209.

Choe, Y., Schuett, M. A., \& Sim, K. W. (2017). An analysis of first-time and repeat visitors to Korean national parks from 2007 and 2013. Journal of Mountain Science, 14, 2527-2539.

Choi, K.W., Chen, C.Y., Stein, M.B., Klimentidis, Y.C., Wang, M.J., Koenen, K.C., \& Smoller, J.W. (2019). Assessment of bidirectional relationships between physical activity and depression among adults: a 2-sample mendelian randomization study. JAMA Psychiatry. doi: 10.1001/jamapsychiatry.2018.4175

Coghlan, A. (2015). Tourism and health: using positive psychology principles to maximise participants' wellbeing outcomes-a design concept for charity challenge tourism. Journal of Sustainable Tourism, 23, 382-400.

Corazon, S., Nyed, P., Sidenius, U., Poulsen, D., \& Stigsdotter, U. (2018). A long-term follow-up of the efficacy of nature-based therapy for adults suffering from stress-related illnesses on levels of healthcare consumption and sick-leave absence: a randomized controlled trial. International Journal of Environmental Research and Public Health, 15, 137.

Duvall, J., \& Kaplan, R. (2014). Enhancing the well-being of veterans using extended groupbased nature recreation experiences. Journal of Rehabilitation Research and Development, $51,685-696$.

Dzhambov, A., Hartig, T., Markevych, I., Tilov, B., \& Dimitrova, D. (2018). Urban residential greenspace and mental health in youth: Different approaches to testing multiple pathways yield different conclusions. Environmental Research, 160, 47-59. 
Frieden, T.R. (2017). Evidence for health decision making-beyond randomized, controlled trials. New England Journal of Medicine, 377, 465-475.

Frühauf, A., Niedermeier, M., Elliott, L.R., Ledochowski, L., Marksteiner, J., \& Kopp, M. (2016). Acute effects of outdoor physical activity on affect and psychological well-being in depressed patients: a preliminary study. Mental Health and Physical Activity, 10, 4-9.

Frumkin, H., Bratman, G.N., Breslow, S.J., Cochran, B., Kahn, P.H., Lawler, J.J., et al. (2017). Nature contact and human health: a research agenda. Environmental Health Perspectives, 125, 075001. doi:10.1289/EHP1663

Gottfredson, D.C., Cook, T.D., Gardner, F.E., Gorman-Smith, D., Howe, G.W., Sandler, I.N., $\&$ Zafft, K.M. (2015). Standards of evidence for efficacy, effectiveness, and scale-up research in prevention science: next generation. Prevention Science, 16, 893-926.

Groulx, M., Lemieux, C., Dawson, J., Stewart, E., \& Yudina, O. (2016). Motivations to engage in last chance tourism in the Churchill Wildlife Management Area and Wapusk National Park: the role of place identity and nature relatedness. Journal of Sustainable Tourism, 24, 1523-1540.

Hardiman, N., \& Burgin, S. (2017). Nature tourism trends in Australia with reference to the Greater Blue Mountains World Heritage Area. Journal of Sustainable Tourism, 25, 732-745.

Hensher, D.A., \& Johnson, L.W. (2018). Applied Discrete-Choice Modelling. Routledge, London.

Holland, W.H., Powell, R.B., Thomsen, J.M., \& Monz, C.A. (2018). A systematic review of the psychological, social, and educational outcomes associated with participation in wildland recreational activities. Journal of Outdoor Recreation, Education, and Leadership, 10, 197225.

Howard Centre for Investigative Journalism (2019). Code Red. https://cnsmaryland.org/interactives/summer-2019/code-red/index.html Accessed 16 Jan 2019.

Jerrett, M., \& van den Bosch, M. (2018). Nature exposure gets a boost from a cluster randomized trial on the mental health benefits of greening vacant lots. JAMA Network Open, 1, e180299-e180299.

Kenett, R.S., Pfeffermann, D., \& Steinberg, D.M. (2018). Election polls — a survey, a critique, and proposals. Annual Review of Statistics and Its Application, 5, 1-24.

Kil, N., Holland, S. M., Stein, T. V., \& Ko, Y. J. (2012). Place attachment as a mediator of the relationship between nature-based recreation benefits and future visit intentions. Journal of Sustainable Tourism, 20, 603-626.

Kim, H., Lee, S., Uysal, M., Kim, J., \& Ahn, K. (2015). Nature-based tourism: Motivation and subjective well-being. Journal of Travel \& Tourism Marketing, 32, S76-S96. 
Larson, L. R., Jennings, V., \& Cloutier, S. A. (2016). Public parks and wellbeing in urban areas of the United States. PLoS One, 11, e0153211.

Lee, B.Y., Adam, A., Zenkov, E., Hertenstein, D., Ferguson, M.C., Wang, P.I., et al. (2017). Modeling the economic and health impact of increasing children's physical activity in the United States. Health Affairs, 36, 902-908.

Lengieza, M.L., Hunt, C.A., \& Swim, J.K. (2019). Measuring eudaimonic travel experiences. Annals of Tourism Research, 74, 195-197.

Levi, E., Dolev, T., Collins-Kreiner, N., \& Zilcha-Mano, S. (2018). Tourism and depressive symptoms. Annals of Tourism Research, 74, 191-194

McDaid, D., Park, A. L., \& Wahlbeck, K. (2019). The economic case for the prevention of mental illness. Annual Review of Public Health, 40, 1-17

Mackenzie, S.H., \& Kerr, J.H. (2013). Stress and emotions at work: An adventure tourism guide's experiences. Tourism Management, 36, 3-14.

Moal-Ulvoas, G. (2017). Positive emotions and spirituality in older travelers. Annals of Tourism Research, 66, 151-158.

Ohe, Y., Ikei, H., Song, C., \& Miyazaki, Y. (2017). Evaluating the relaxation effects of emerging forest-therapy tourism: A multidisciplinary approach. Tourism Management, 62, 322-334.

Patel, V., Saxena, S., Lund, C., Thornicroft, G., Baingana, F., Bolton, P., et al. (2018). The Lancet Commission on Global Mental Health and Sustainable Development. The Lancet doi: 10.1016/S0140-6736(18)31612-X

Propper, C., Jones, K., Bolster, A., Burgess, S., Johnston, R., \& Sarker, R. (2005). Local neighbourhood and mental health: evidence from the UK. Social Science \& Medicine, 61, 2065-2083.

Puhakka, R., Pitkänen, K., \& Siikamäki, P. (2017). The health and well-being impacts of protected areas in Finland. Journal of Sustainable Tourism, 25, 1830-1847.

Ramkissoon, H., Smith, L.D.G, \& Weiler, B. (2013). Relationships between place attachment, place satisfaction and pro-environmental behaviour in an Australian national park. Journal of Sustainable Tourism, 21, 434-457.

Shanahan, D.F., Bush, R., Gaston, K.J., Lin, B.B., Dean, J., Barber, E., et al. (2016). Health benefits from nature experiences depend on dose. Scientific Reports, 6, 28551

Smith, M.K., \& Diekmann, A. (2017). Tourism and wellbeing. Annals of Tourism Research, $66,1-13$.

Song, J., Fry, R., Mizen, A., Akbari, A., Wheeler, B., White, J., et al. (2018). Association between blue and green space availability with mental health and wellbeing. International Journal of Population Data Science, 3, 330. 
Stafford, M., Gale, C.R., Mishra, G., Richards, M., Black, S., \& Kuh, D.I. (2015). Childhood environment and mental wellbeing at age 60-64 years: prospective evidence from the MRC National Survey of Health and Development. PloS ONE 10, e0126683 (2015).

Stern, P.N., \& Porr, C.J. 2017. Essentials of Accessible Grounded Theory. Oxford: Routledge.

Stigsdotter, U.K., Corazon, S.S., Sidenius, U., Nyed, P.K., Larsen, H.B., \& Fjorback, L.O. (2018). Efficacy of nature-based therapy for individuals with stress-related illnesses: randomised controlled trial. British Journal of Psychiatry, 213, 404-411.

Stone, L. S., \& Stone, T. M. (2017). Domestic tourism in Botswana: an examination of nature-based tourism constraints. Journal of Sustainable Tourism, 10, 97-116.

Terraube, J., Fernández-Llamazares, Á., \& Cabeza, M. (2017). The role of protected areas in supporting human health: a call to broaden the assessment of conservation outcomes. Current Opinion in Environmental Sustainability, 25, 50-58.

Thomsen, J.M., Powell, R.B., \& Monz, C.A. (2018) A systematic review of the physical and mental health benefits of wildland recreation. Journal of Parks and Recreation

Administration, 36, 123-148.

Tullett, A.M., \& Plaks, J.E. (2016). Testing the link between empathy and lay theories of happiness. Personality and Social Psychology Bulletin, 42, 1505-1521.

Twohig-Bennett, C., \& Jones, A. (2018). The health benefits of the great outdoors: a systematic review and meta-analysis of greenspace exposure and health outcomes. Environmental Research, 166, 628-637.

Uysal, M., Sirgy, M.J., Woo, E., \& Kim, H.L. (2016). Quality of life (QOL) and well-being research in tourism. Tourism Management, 53, 244-261.

Veal, A.J. (2017). Research Methods for Leisure and Tourism. London: Pearson.

Wang, R.A.H., Nelson-Coffey, S.K., Layous, K., Bao, K.J., Davis, O.S., \& Haworth, C.M., (2017). Moderators of wellbeing interventions: why do some people respond more positively than others? PLoS One, 12, e0187601.

Warner, R.P., Meerts-Brandsma, L., \& Rose, J. (2020). Neoliberal ideologies in outdoor adventure education: barriers to social justice and strategies for change. Journal of Park and Recreation Administration, in press.

Weaver, D.B., \& Lawton, L.J. (2002). Overnight ecotourist market segmentation in the Gold Coast hinterland of Australia. Journal of Travel Research, 40, 270-280.

White, M.P., Elliott, L.R., Taylor, T., Wheeler, B.W., Spencer, A., Bone, A., et al. (2016). Recreational physical activity in natural environments and implications for health: a population based cross-sectional study in England." Preventive Medicine, 91, 383-388. 
White, M.P., Alcock, I., Grellier, J., Wheeler, B.W., Hartig, T., Warber, S.L., et al. (2019). Spending at least 120 minutes a week in nature is associated with good health and wellbeing. Scientific Reports, 9, 7730.

Wolf, I. D., Ainsworth, G. B., \& Crowley, J. (2017). Transformative travel as a sustainable market niche for protected areas: a new development, marketing and conservation model. Journal of Sustainable Tourism, 25, 1650-1673.

Wolf, I. D., Stricker, H. K., \& Hagenloh, G. (2015). Outcome-focused national park experience management: Transforming participants, promoting social well-being, and fostering place attachment. Journal of Sustainable Tourism, 23, 358-381.

Wyles, K.J., White, M.P., Hattam, C., Pahl, S., King, H., \& Austen, M. (2017). Are some natural environments more psychologically beneficial than others? The importance of type and quality on connectedness to nature and psychological restoration. Environment and Behaviour doi:10.1177/0013916517738312

Xiao, L.L., Zhong, L.S., Zhou, R., and Yu, H. (2017). Review of international research on national parks as an evolving knowledge domain in recent 30 years. Progress in Geography, $36,244-255$.

Xu, H., Cui, Q., Sofield, T., and Li, F.M.S. (2014). Attaining harmony: understanding the relationship between ecotourism and protected areas in China. Journal of Sustainable Tourism, 22, 1131-1150.

Young, J., McGrath, R., \& Adams, C. (2018). Fresh air, sunshine and happiness: millennials building health (salutogenesis) in leisure and nature. Annals of Leisure Research, 21, 324346.

Zanon, D., Curtis, J., Lockstone-Binney, L., \& Hall, J. (2019). Examining future park recreation activities and barriers relative to societal trends. Annals of Leisure Research, 22, 506-531. 
Table 1. Thematic Analysis, Q1: General Motivations to Visit Forest or Beach Parks

\begin{tabular}{|c|c|c|}
\hline Theme, construct & Concepts, beach parks & Concepts, forest parks \\
\hline \multirow[t]{2}{*}{ Beauty, scenery } & Ocean so blue/green/clean & Cliffs, creeks, mist, sun after rain \\
\hline & Breathe the ocean air & Always smells good/clean \\
\hline \multirow[t]{2}{*}{ Natural history } & $\begin{array}{l}\text { Look for whales/dolphins/turtles } \\
\text { [worried about jellyfish/sharks] }\end{array}$ & $\begin{array}{l}\text { Birds/wallabies/flowers/fungi/ } \\
\text { butterflies/crayfish }\end{array}$ \\
\hline & Photography, binoculars & Photography, binoculars \\
\hline Appreciation & Lucky we have beach/park & Lucky the park/forest is so near \\
\hline Activities, exercise & $\begin{array}{l}\text { Swim/surf/run/bike/yoga/fitness/ } \\
\text { walk dog }\end{array}$ & $\begin{array}{l}\text { Hike, walk, run (often mention } \\
\text { distance or name of trail) }\end{array}$ \\
\hline \multirow[t]{3}{*}{ Escape, relax } & Get out of house/office/city & So different from the city \\
\hline & Sunbathe, sleep on beach, read & Calm, peaceful, birdsong, frogs \\
\hline & Collect my thoughts & Stop thinking about work \\
\hline \multirow[t]{4}{*}{ Family, friends } & Kids enjoy it & Family visits, camping \\
\hline & [kids tired/sunburnt/hungry] & [kids tired/wet/hungry] \\
\hline & Meet up, social group & Walking groups and clubs \\
\hline & WoM/social media & WoM/social media \\
\hline
\end{tabular}

Table 2. Q2: Emotions, Recovery, Worldview

\begin{tabular}{|l|r|r|r|}
\hline Question asked (total n=238) & Total & +ve & $\mathbf{\% +}$ \\
\hline & & & \\
\hline Emotion, happiness & 112 & 98 & 87.5 \\
\hline Recovery, de-stress & 62 & 37 & 59.7 \\
\hline Worldview & 64 & 13 & 20.3 \\
\hline
\end{tabular}

Table 3. Q3: Direction of Causation

\begin{tabular}{|l|l|r|r|}
\hline & Direction of causation $(\mathbf{n = 9 8 )}$ & \# & \% \\
\hline & & & \\
\hline 1 & Outdoor nature tourism and recreation makes me happier & 64 & 65.3 \\
\hline 2 & When I'm happy I take part in outdoor nature tourism and recreation & 14 & 14.3 \\
\hline 3 & It works both ways, or sometimes one, sometimes the other & 9 & 9.2 \\
\hline 4 & Don't know, can't tell, not sure & 4 & 4.1 \\
\hline 5 & Other specific response & 7 & 7.1 \\
\hline
\end{tabular}

